\title{
SERVICIOS AMBIENTALES: ENTRE LA CONSERVA- CIÓN Y EL USO DE LOS RECURSOS
}

\section{ENVIRONMENTAL SERVICES: BETWEEN CONSERVATION AND USE OF RESOURCES}

https://doi.org/10.38147/invneg.v14i23.135

S. Marcelo Olivera Villarroel.

Profesor Investigador de la Universidad Autónoma Metropolitana, Departamento de Teoría y Procesos del Diseño;

satzcha@msn.com

Ricardo Hernández Murillo.

Investigador Sénior Banco Mundial - México;

hmurillo@economia.unam.mx

Recibido: 08 de marzo de 2021

Aprobado: 15 de abril 2021

\section{Resumen}

El estudio aborda la temática de los servicios ambientales desde una perspectiva de prestación de bienes y servicios públicos, los cuales deben estar normados y reglamentos por el Estado. Para ello se realiza una aproximación cuantitativa que da un contexto a la recomendación de política pública propuesta. Las recomendaciones de política pública tienen como eje central la conservación de coberturas vegetales que prestan servicios ambientales a la sociedad.

La conservación del territorio, a través del pago del servicio ambiental es una política que atraviesa en forma transversal no sólo el contexto ambiental, sino también el contexto social; ya que se reconoce el aporte al sistema productivo de los esfuerzos de conservación y manejo de recursos de las comunidades campesinas y de los sistemas de áreas protegidas nacionales, departamentales y municipales.

\section{Palabras clave.}

Bolivia, pagos, recomendaciones de política pública, servicios ambientales, tenencia de la tierra.

\begin{abstract}
.
The study addresses the issue of environmental services from a perspective of the provision of public goods and services, which should be normed and regulated by the State. To do this, a quantitative approach is carried out that gives context to the recommendation of the proposed public policy. The central policy of the recommendations of public policy is the conservation of vegetables and environmental services in society.

The conservation of the territory, through the payment of the environmental service is a policy that cuts across not only the environmental context, but also the social context; since it is recognized the contribution to the system product of the conservation efforts and the management of the resources of the peasant communities and the systems of national, departmental and municipal protected areas.
\end{abstract}

\section{Keywords.}

Bolivia, environmental services, land tenure, payments, public policy recommendations.

\section{Servicios Ambientales:}

Entre la Conservación y el Uso de los Recursos.

\section{Recomendaciones de Política Pública.}




\section{Investigación Negocios}

\section{Introducción.-}

El reconocimiento de los servicios que brinda el sistema natural a la existencia del planeta, tal como lo conocemos, se manifiesta en los múltiples esfuerzos que se generan en el mundo para su conservación y manejo. Uno de los esfuerzos que mayor empuje ha recibido en los últimos años es el pago de servicios ambientales por conservación de los sistemas forestales (Pagiola et al, 2003; Carvajal et al, 2019). Ello lleva a reconocer en forma implícita la importancia que tiene el bosque en la generación de servicios ambientales, aun cuando no existan investigaciones que corroboren en forma explícita dicha relación (Carvajal et al, 2019)

Dada la participación relativamente pequeña en la generación de empleo y en el ingreso nacional los responsables de las decisiones nacionales asignan baja prioridad a la actividad de conservación y manejo forestal en comparación con otros sectores que compiten por los limitados presupuestos, incluso en el sector ambiental. (FAO, 2015a). Por ello se ha impulsado la creación de mecanismos financieros innovadores como el pago de servicios ambientales por la generación de recursos hídricos.

Es justamente el uso del agua, como principal elemento e insumo de la actividad humana, el que ha generado interés en conservar las fuentes de las que se provee la economía de este vital elemento. Pero éste es sólo uno de los servicios que provee el entorno natural a la actividad humana y analizarlo por separado destruye la concepción del ecosistema que engloba en sí el manejo y conservación de los sistemas naturales y en especial los sistemas forestales.

Los servicios generados por un sistema forestal o cualquier entorno natural son parte de un sistema en el cual la interrelación y dependencia de las partes es latente; por lo que las políticas de administración deben ir al manejo de todo el entorno y no sólo de una de sus partes (Landell-Mills \& Porras, 2002)'. Adicionalmente al manejo integral de todos los servicios prestados por un entorno natural, se debe incluir a los habitantes de este sistema no sólo como usuarios; sino como parte del mismo sistema (Rosas-Baños, 2013). ${ }^{2}$

Este reconocimiento de la integralidad de los servicios ambientales, debe hacerse tanto desde una perspectiva ecológica, económica, social y desde un enfoque de administración del recurso; ya que de hecho al pagar por un servicio, por ejemplo la biodiversidad, estamos conservando el bosque en forma integral. De otra forma se estaría recargando los costos de manejo de todos los servicios a solamente un grupo de usuarios, en el caso del pago por servicios ambiéntales hídricos, a los potenciales usuarios del servicio agua.

Los paisajes rurales contienen existencias (stock) y generan flujos (flow) que son aprovechados por la población en su existencia y en sus actividades productivas. Estos activos y servicios ambientales proporcionan beneficios a la población en un área de influencia mayor a la de su entorno paisajístico. Las ciencias físicas nos ayudan a comprender las existencias y flujos de energía; en tanto que la economía da cuenta de ellos como externalidades positivas, que aportan beneficios a las actividades económicas fuera del área.

Las ciencias de la tierra aportan crecientes evidencias de la dinámica de los servicios ambientales en el paisaje rural, así como indicaciones cada vez mayores de las lagunas en nuestro conocimiento sobre los procesos de generación de los mismos. La investigación de campo combinada, síntesis de más de 600 observaciones, los modelos climáticos y económicos documentan pérdidas sustanciales en el flujo de la corriente y un aumento de la salinización y acidificación del suelo, con aforestación. Las plantaciones disminuyeron el flujo de la corriente en 227 milímetros por año a nivel mundial (52\%), y el 13\% de las corrientes se secaron completamente durante al menos 1 año. El modelado regional de los escenarios de plantación en los Estados Unidos sugiere que es poco probable que las retroalimentaciones climáticas compensen tales pérdidas de agua y puedan exacerbarlas. Las plantaciones pueden ayudar a controlar la recarga de aguas subterráneas y los afloramientos, pero reducen el flujo de la corriente y salinizan y acidifican algunos suelos. (Jackson et al., 2005)

$1 \quad$ Al crear un mercado para un servicio ambiental, este servicio tenderá a beneficiarse de nuevos flujos de inversión y menor negligencia. Pero, aquellos servicios que permanecen fuera del sistema de mercado no necesariamente se benefician e incluso pueden verse afectados negativamente por la inversión canalizada hacia el servicio recién comercializado.

$2 \quad$ Las reglas de aplicación de los mecanismos de compensación determinan en gran medida su capacidad de inclusión. Si las reglas no se diseñan deliberadamente para favorecer a las comunidades rurales pobres, los instrumentos de compensación pueden generar mayor inequidad y exclusión social. 
La investigación, en este sentido, utilizará como métodos de análisis a la economía ambiental, la ecología ${ }^{3}$ y a los procesos de organización social, por ello se emplearán herramientas de estas disciplinas, partiendo en la división de los servicios ambientales entre demandantes y oferentes de los mismos, pero a partir de los sistemas y coberturas vegetales existentes en un entorno.

De este análisis, se derivarán recomendaciones de política pública, las cuales tendrán como eje central la conservación de coberturas vegetales que presten servicios ambientales a la sociedad en su conjunto. La conservación del territorio, a través del pago del servicio prestado es una política que atraviesa en forma transversal no sólo el contexto ambiental, sino también el contexto social al reconocer la importancia dentro el sistema productivo de las comunidades campesinas y de los sistemas de áreas protegidas nacionales, departamentales y municipales.

\section{Oferta de servicios ambientales. EI costo de oportunidad y la disposición a aceptar un pago por parte de los pro- pietarios y/o administradores del bos- que}

La caracterización de los servicios ambientales en Bolivia parte por diferenciar tres grandes zonas que tienen coberturas de suelo y procesos diferenciados de manejo entre sí, la primera la zona andina, la segunda la región sub-andina y la tercera los grandes llanos amazónicos y chaqueños. ${ }^{4}$ Estas zonas no sólo se diferencian en su tipo de cobertura sino también en su sistema de administración y uso del suelo determinado por su contexto histórico y social. ${ }^{5}$

Cada una de estas regiones tiene sus propias características biogeográficas con un conjunto de flujos de servicios ambientales provenientes de ellas, por ejemplo: la dotación de fuentes de agua, flujo estable de lluvias, composición del suelo, tipo de cobertura vegetal, etc. A partir de estas características y servicios la sociedad decide la función que se le dará a su entorno entre usos urbanos, agrícolas, ganaderos, forestales y de conservación entre otros.

Este cambio en el uso parte en gran medida de las demandas de la sociedad en la generación de bienes y servicios para su subsistencia y crecimiento. La toma de decisiones para este proceso de cambio no es fácil e incluye no sólo una decisión económica, sino social y cultural. Lamentablemente al considerar a los servicios ambientales como bienes de libre acceso y no reconocer las externalidades positivas brindadas por el ambiente, nuestra sociedad ha venido destruyendo en forma sostenida justamente las características que llevaron a usar estas áreas para cada una de las funciones que viene desarrollando para la sociedad.

La mayor presión de cambio de uso del suelo, suele estar en la conversión de áreas de uso agrícola de alta calidad a urbano con el consecuente aumento de la presión en habilitar nuevas áreas de cultivo con la sustitución de áreas forestales $u$ otro tipo de suelos no tan apropiados para la agricultura. Esto lleva a perder importantes extensiones de superficie de suelo con coberturas vegetales que brindan otro tipo de servicios ambientales que en última instancia son más valorados por la economía que el uso agrícola o ganadero. Ver cuadro $\mathrm{N}^{\circ} 5$ en anexos.

\subsection{La distribución de tierras en Bolivia}

La actual distribución de tierras en Bolivia surge del proceso de reforma agraria iniciado a partir de principios de $1950^{6}$. Hasta 1992, el proceso de reforma repartió en forma legal el $43 \%$ del territorio boliviano a aproximadamente 620.000 beneficiarios, pero al existir un proceso indebido de apropiación de tierras en especial en la región oriental del país, se decide cambiar el sistema de reparto e iniciar un proceso de saneamiento de títulos mediante

3 Se usará una aproximación ecosistémica del paisaje: la tierra es un factor dinámico no fijo, cambia y se modifica en el tiempo

$4 \quad$ Bolivia cuenta con aproximadamente 17 eco-regiones cada una con características muy particulares de biodiversidad y cobertura vegetal. Cada una de estas regiones cumple un importante papel en el equilibrio ecológico del país e interactúan una con la otra

$5 \quad$ La región andina está caracterizada por la presencia de grupos indígenas que aun mantienen su sistema de organización precolombino, sistemas de ayllus entre otros; en la región de los llanos existen grupos amazónicos tribales que tienen el manejo de sus sistemas forestales, a través de los Territorios Comunitarios de Origen- TCO 's; mientras que en parte de las regiones andinas, sub-andina y de los llanos existe un sistema de tenencia de la tierra en función a la propiedad privada fruto de la reforma agraria acaecida en la década de los años 50 en Bolivia.

$6 \quad$ La máxima de este proceso puede expresarse en la siguiente frase, "la tierra es para quien la trabaja" 


\section{Investigación Negocios}

la promulgación de la ley Nº 1715 del Servicio Nacional de Reforma Agraria el año 1996. (Sanjines, 2005)

Este proceso de saneamiento de tierras, que aún se encuentra en proceso, se puede resumir en el siguiente cuadro de sus avances más notables:

\begin{tabular}{|l|c|c|}
\hline \multicolumn{3}{|c|}{ Cuadro $\mathbf{N}^{\circ} \mathbf{1}$} \\
\hline $\begin{array}{l}\mid \text { Estado del proceso de saneamiento de tierras } \\
\text { a nivel nacional }\end{array}$ \\
\hline Categoría & $\begin{array}{l}\text { Categoría en } \\
\text { Has. }\end{array}$ & $\%$ \\
\hline Superficie saneada & 14078866 & $13 \%$ \\
\hline Superficie en proceso & 37183670 & $35 \%$ \\
\hline Superficie por sanear & 56001558 & $52 \%$ \\
\hline Total & $\mathbf{1 0 7 2 6 4 0 9 4}$ & $\mathbf{1 0 0} \%$ \\
\hline Fuente: INRA 2014 & \\
\hline
\end{tabular}

Como se ve más del 52 \% del territorio no cuenta con su debida titulación, por lo que el estatus legal de estos territorios aún está en entredicho. Muchos de estos territorios pertenecen al sistema nacional de áreas protegidas (20\% del territorio), a comunidades indígenas de la región amazónica y de la región andina del país.

\subsection{El Pago de Servicios Ambiéntales}

El pago por servicios ambientales -PSA- consiste en el proceso de compensación directa a los propietarios, administradores o usuarios de la tierra por los servicios ambientales que producen (Pagiola \& Landell-Mills, 2003). De esta manera se generan incentivos directos para que los propietarios o usuarios de la tierra incluyan estos servicios en sus decisiones sobre los usos del suelo. Tanto Costa Rica ${ }^{7}$ como México, entre otros han explorado este tipo de pagos para la mejor conservación de sus coberturas forestales y el mantenimiento de sus sistemas y entornos naturales.

Los métodos de pago, con más de 800 casos documentados a la fecha a nivel mundial, incluyen desde acuerdos cooperativos intra e intercomunitarios, hasta verdaderos mercados de derivados como es el caso del Mecanismo de Desarrollo Limpio de la Convención Marco de las Naciones Unidas sobre Cambio Climático. La dificultad inherente a la medición de "intangibles" como se ha llamado a las externalidades positivas del bosque, ha llevado a las diferentes partes contratantes a adoptar diversos proxys para poder evaluar el cumplimiento de los acuerdos.

En los acuerdos bilaterales locales, el "comprador" usualmente tiene una idea de las conductas de manejo que desea modificar o incentivar, así como de las manifestaciones objetivas de las mismas. Aún en casos más complejos como el de Catskills, la formulación de un plan de manejo por granja, ha permitido identificar acciones y parámetros específicos como la construcción de un puente para canalizar el paso del ganado sin afectar el curso del arroyo.

En programas masivos como las convocatorias de pago de servicios ambientales operadas por las autoridades forestales, la simple conservación de un parámetro como el porcentaje de cobertura forestal, es convenido como el indicador síntesis de control de plagas, incendios e ilícitos, indispensables para la conservación del bosque. En los proyectos de biodiversidad suelen adoptarse especies indicadoras que, de la misma manera que la cobertura de copa, puedan resumir una serie de procesos que estaría garantizando la conservación de dicha especie.

$7 \quad$ Los arreglos contractuales bipartitos entre actores interesados en la conservación del bosque por los servicios ambientales que presta no son algo nuevo. La preocupación por la calidad ha sido también móvil para que empresas hidroeléctricas realicen pagos a los propietarios de los bosques en sus cuencas de abastecimiento, y en algún caso, este pago por servicios de cuenca ha sido un instrumento para sortear un conflicto de tierras garantizando el acceso al agua por parte de la hidroeléctrica y recursos para sostener la conservación en el caso de la asociación civil responsable de la reserva. 
Particulares, asociaciones, empresas, gobiernos locales y nacionales han promovido esquemas, con aproximaciones diversas por cuanto a la asignación de derechos y el tratamiento de los servicios como bienes públicos o privados, pero el común denominador lo constituye la dificultad para definir el objeto preciso de la transacción, la expresión medible del servicio.

Dos corrientes de pensamiento se enfrentan en la práctica cotidiana: a) la que sostiene la relevancia social de la conservación de los bosques y la disposición a pagar de la sociedad que explica la existencia de acuerdos bilaterales y programas públicos, tarifas, subsidios, inversiones y transferencias condicionadas a la conservación de la fuente de los servicios ambientales; y b) los que quisieran condicionar la acción social a una certeza científica basada en medios de medición de los servicios ambientales cuya complejidad hace difícil ajustar la realidad a un modelo simple donde exista un productor y un usuario y en el que sus acuerdos estén condicionados al monitoreo del cumplimiento de parámetros precisos que se basarían en un conocimiento científico siempre en construcción.

En el primer caso estamos asistiendo a un tratamiento de "bien público" donde la sociedad está más interesada en la preservación del capital natural y el servicio ambiental -consiente del costo para la sociedad en caso de perderles-, y la necesidad de construir las instituciones (acuerdos sociales y reglas) que garanticen la disposición a pagar y le exclusión del free rider dada la dificultad de exclusión intrínseca a las externalidades positivas generadas por los ecosistemas forestales. Sin ignorar la necesidad de avanzar en la asignación eficiente de los costos y los beneficios de su cuidado, esta corriente alerta sobre los riesgos de pretender lograrlo con base en modelos limitados a las variables conocidas y medibles que no alcanzan a capturar las dinámicas de estos sistemas complejos. En el segundo caso, los estudiosos (y algunos detractores) ignoran la dimensión estratégica de un fenómeno social como la emergencia del mercado de servicios ambientales, convencidos de que sólo el conocimiento positivo de las variables de un modelo lineal de causa-efecto que explique las condiciones antropogénicas para la provisión de un servicio (y la evidencia para "convencer" a un comprador potencial) permitirá generar un mercado que garantice no sólo su permanencia sino la mayor eficiencia en la asignación de costos y beneficios como si el objetivo se limitase a la generación de bienes privados (productos), cuando el reto radica precisamente en la preservación del complejo sistema de stocks y flujos que genera los servicios eco-sistémicos valorados por la sociedad.

\title{
La decisión del propietario de conservar el bosque, mejorar su manejo o cambiar el uso del suelo.
}

\author{
Etapas básicas en la decisión del propietario del bosque \\ ૫ Identificación de los conjuntos de oportunidades \\ $\checkmark$ Definición de las disyuntivas \\ \ Cálculo correcto de los costos: oportunidad, irrecuperables, marginales
}

El conjunto de oportunidades muestra cuáles son las opciones que tiene el propietario de la tierra, independientemente de que éste sea un pequeño, un gran propietario o el Estado mismo. Cada uno de estos actores tendrá preferencias, restricciones presupuestarias y de tiempo que limitan el conjunto de opciones reales para cada actor. Aún dentro de las restricciones puede existir una fuerte disyuntiva entre las opciones reales, y lo que habrá de aportar el cálculo correcto de los costos es una ponderación objetiva al momento de analizar las opciones.

\subsubsection{El Costo de Oportunidad}

El costo de oportunidad es el costo de utilizar un recurso cualquiera, midiéndolo comparativamente con el siguiente mejor uso que podría dársele. Un costo irrecuperable es un gasto realizado en el pasado y que no podrá recuperarse, independientemente de la decisión que se tome en el momento presente. Es una referencia útil, pero difícilmente será racional factorizarlo en la decisión presente. Finalmente, el costo marginal es aquel en que incurrimos para llevar una situación más allá de su estatus presente, donde cada beneficio adicional (o marginal) implicará un costo marginal.

La introducción de un Pago por Servicios Ambientales introduce un elemento importante en 


\title{
184 Investigación \&Negocios
}

la decisión del propietario del bosque: la decisión de "aceptar" este pago dependerá de cómo este uso (conservación y/o buen manejo para la producción de servicios ambientales) se mida comparativamente con el siguiente mejor uso que podría dársele al recurso forestal. Igualmente, a partir de que se ha instituido el PSA, éste se convierte en un "siguiente mejor uso" contra el que habrá de medirse cualquier opción de uso futuro o potencial.

En las decisiones de política para reconocer las externalidades y encarar el reto de la preservación de los servicios ambientales del bosque, se juega una apuesta clave: la equidad. Coase (1960) preveía que una vez asignados los derechos de propiedad, las soluciones de mercado o la negociación entre los usuarios potenciales garantizarían resultados eficientes, pero en las decisiones de política no pueden ignorarse los impactos distributivos de las decisiones, (Mabsout \& Radmard, 2019).

Ciertamente en los casos revisados por la literatura económica destaca la importancia de las instituciones y la asignación de derechos de propiedad en la definición de las políticas adoptadas, y éstas reflejan la diversidad anticipada por Coase, quien sostenía no sólo que la asignación de derechos de propiedad garantiza un resultado eficiente, sino que la forma de asignación de los derechos de propiedad afectará la distribución de la renta, y la inequidad no contribuye a la eficiencia económica. Ver cuadro $\mathrm{N}^{\circ}$ en anexos

\subsubsection{Certezas de mercado y cos- tos de oportunidad}

Por la naturaleza del stock de capital natural requerido para garantizar los flujos de servicios ambientales, los arreglos contractuales tienen que ser de mediano y preferentemente, de largo plazo. La declaratoria de áreas naturales protegidas, las servidumbres ecológicas o land easements, y los contratos de adhesión a programas de compensación por los servicios ambientales del bosque y la conser-

vación de las cuencas hidrológicas son todos instrumentos para asegurar la base natural de una actividad económica, a largo plazo.

Por otra parte, el entorno económico que ha caracterizado la producción agropecuaria, - principal causa de cambio de uso de suelo forestal en los años 60 y $70^{8}$-, especialmente en los últimos 30 años, es el de una gran incertidumbre: a) terminó la revolución industrial, el Estado ha dejado de proveer insumos, crédito y extensión rural, ya no hay precios de garantía y subsidios; y b). La apertura comercial enfrenta a los productores rurales con la competencia subvencionada de Norteamérica y Europa. La actividad agropecuaria se ha convertido, al menos por el momento, en una actividad de alto riesgo que demanda cada vez más insumos y capital, y que ofrece cada vez menores márgenes de utilidad con menores certezas de generación de ingresos.

El propietario de la tierra puede estar convencido de que vivimos un proceso de revalorización de los recursos naturales planetarios, seguramente comparte la visión de Mark Twain quien aconsejaba: "hijo, compra tierra, ya no están haciendo más!" Pero lo cierto es que el mercado hoy aún no lo premia. El retorno de su actividad productiva no se lo reconoce, pero el propietario rural se aferra a la tierra por razones culturales o porque intuye el valor futuro de su activo. En este contexto, puede resultar atractivo un instrumento de mediano o largo plazo que le garantiza una renta que, aunque modesta, le permite: a) minimizar su riesgo presente, y b) conservar su valor de opción.

Además de la certidumbre estrictamente económica, los arreglos institucionales que promueven el Pago de Servicios Ambientales, -explícita o implícitamente, constituyen también un reconocimiento de derechos sobre los servicios ambientales y por ende sobre la propiedad de la tierra y los recursos biológicos que los producen (Muñoz et al., 2005). ${ }^{9}$

\subsubsection{Derechos de propiedad}

Además de la condición ex ante propuesta

\begin{abstract}
8 Aun cuando la estadística de pérdida de bosques en América Latina muestra que la mayor proporción de hectáreas forestales fueron perdidas frente a los usos ganaderos y agrícolas, en las últimas décadas se atribuye al crecimiento de las ciudades y la infraestructura, los enclaves mineros, industriales y turísticos, el abandono de tierras que las expone a plagas, incendios y talla ilegal, un importante impacto ambiental. Ciertamente, es difícil conservar tierras y bosques cuando las alternativas de uso alcanzan un gran valor por su vinculación a centros urbanos o actividades económicas relevantes.

$9 \quad$ Los programas de PSA no son la panacea. Simplemente son una adición nueva y valiosa al conjunto de políticas disponibles para resolver tanto la deforestación como los problemas relacionados con el agua, uno en el que las fallas del mercado se corrigen de manera directa y el otro, que define los derechos de propiedad sobre los servicios ambientales a favor de los propietarios de bosques.
\end{abstract}


por Coase para asegurar el intercambio y la asignación eficiente a través del mercado, la asignación de derechos de propiedad es el mayor reto social, legal y económico en el caso de los servicios ambientales. La posibilidad de reivindicación de los derechos de propiedad y la capacidad de exclusión es, en efecto, más sencilla en algunos bienes: históricamente en los bienes producidos por el hombre-artesano. La mayor dificultad de reivindicación de los derechos de propiedad y la exclusión de sus beneficios a "polizones" o free riders, ha dependido siempre de la construcción de instituciones que regulan con legitimidad y eficacia.

Un bien tan "intangible" como la contribución a la regulación climática a través de la función de "sumidero" de carbono que ejercen los bosques, ha sido estríctamente regulada por el Mecanismo de Desarrollo Limpio, demostrando de manera paradigmática, cómo la construcción institucional puede manejar eficazmente la dificultad de exclusión presente en estos bienes.

Para comprender el proceso de apropiación/ asignación y posterior compromiso de los derechos sobre los bienes y servicios ambientales, es útil desagregarlos de la siguiente manera (Rosa et al, 2004):

- Acceso: Derecho de entrar a un espacio físico definido y disfrutar beneficios no-extractivos, como actividades de recreación.

- Retiro: Derecho de extraer recursos o productos de un sistema (p.e. pesca, leña, agua para riego o consumo humano, etc.).

- Manejo: Derecho de regular los patrones de uso interno y transformar el recurso.

- Exclusión: Derecho de determinar quién tiene derechos de acceso, retiro, manejo, y cómo estos derechos se transfieren.

- Enajenación: Derecho de traspasar los derechos de manejo y exclusión.
De la revisión de casos se puede concluir que los principales actores afectados por los esquemas de PSA son los propietarios o poseedores del bosque.

Para los propietarios de los recursos naturales, las implicaciones de los esquemas de Pago de Servicios Ambientales identificadas incluyen (Madrid, 2016):

- Cambio en la propiedad del terreno

- Restricción total o parcial en el acceso y uso de los espacios y recursos biológicos

- Cambios en las prácticas de manejo

- Sin cambios

Con respecto a los impactos para los usuarios de los servicios ambientales, los dos casos que son sitio-dependientes en un mayor grado, son los usuarios del agua y los de la belleza escénica. Especialmente en el primer caso, la dependencia de una sola cuenca como fuente de abastecimiento suele ser técnica o económicamente alta. Aún en este caso, el impacto directo del manejo del bosque sobre la disponibilidad, estacionalidad o calidad del agua no está suficientemente establecida (Jackson et al., 2005), debido a que la evidencia empírica no reporta impactos significativos inmediatos, por lo que la presión para el usuario se basa en una convención social (y en el imperativo económico de la percepción de escasez presente o futura), antes que en una evidencia directa causa-efecto.

\subsection{Beneficios sociales y ambientales: Exclusión, Pobreza y Conservación}

En términos de la pobreza rural, debe contemplarse el papel de los productos y servicios de la naturaleza como un recurso que puede revalorarse y convertirse en un activo. Los casos de Costa Rica (Miranda, 2003) ${ }^{10}$ y la India nos ofrecen ejemplos en los que un Programa de Pago de Servicios Ambientales ha sido diseñado expresamente para ser in-

\footnotetext{
$10 \quad$ Si bien el esquema de PSA no es un programa de bienestar social, desde el principio, el estado y varias organizaciones sociales asumieron que contribuiría al alivio de la pobreza rural en Costa Rica. Sin embargo, tiene un impacto significativo en el presupuesto familiar. El impacto principal en los activos humanos se relaciona con el desarrollo de capacidades a diferentes niveles, ha habido una mejora sustancial en la educación ambiental y la gestión de residuos sólidos, que involucra a escuelas, padres y la sociedad civil.
}

ISSN ONLINE 2521-2737|REV.INV.\&NEG.|V.14| № 23|AÑO 2021 


\section{Investigación $\&$ Negocios}

cluyente y reconocer derechos a los "cuidadores" de los recursos naturales responsables de la conservación y el manejo que produce los servicios ambientales.

El reconocimiento del papel fundamental de los servicios ambientales en la economía y en el sostenimiento de la vida en general, ofrece una plataforma apropiada para promover el reconocimiento de los servicios y bienes públicos y la regulación que permita fortalecer mecanismos e instrumentos para garantizar estos bienes y servicios ambientales

\section{Demanda de Servicios Ambientales. La Disposición a Pagar por el Cambio en el Uso del Suelo}

Se puede decir que todos los habitantes de la tierra somos demandantes de servicios ambientales, pudiendo demandar servicios desde una perspectiva local, regional o global. Los servicios ambientales en la mayoría de los casos son de hecho un bien público, por lo que hay que tratarlos como tal. Las definiciones clásicas de los bienes y servicios públicos les atribuían como características: la no rivalidad y no exclusión en su consumo" además de ser un bien o servicio de libre acceso ${ }^{12}$ (Azqueta, 2012). En la mayoría de los casos estos bienes y servicios generan algún tipo de externalidad, aunque su manejo y conservación no era reconocido por la sociedad ${ }^{13}$ (Freeman III, 1993). El reconocimiento de la escasez real o futura de los servicios refleja la emergencia

de la rivalidad en un bien que con ser público empieza a mostrar su finitud y el reconocimiento de la complejidad de los ecosistemas forestales que los producen ha llevado a la construcción de instituciones (acuerdos y reglas) que permitan la exclusión y el "pago" a los propietarios o "cuidadores" del bosque.

Esto entraña que el manejo de política de los servicios ambientales sobre todo los considerados servicios públicos debe partir de un sistema de regulación de mercado, esto implica que en su análisis intervienen todas las problemáticas de la dotación de bienes públicos. Esto no excluye la posibilidad de que existan acuerdos entre privados que generen acuerdos de intercambio de servicios por un pago determinado, ciertamente este tipo de transacciones no son la regla; ya que se deben dar condiciones muy especiales para el surgimiento de estos acuerdos. ${ }^{14}$

Para desarrollar el manejo de bienes públicos desde la perspectiva económica, se puede usar la regla de Samuelson ${ }^{15}$ sobre la distribución óptima de bienes públicos. La cual es una construcción teórica general que indica que la sociedad debe brindar en forma óptima un bien público hasta que las sumas de las tasas marginales de sustitución entre el bien público y el resto de los bienes privados de la sociedad iguale la tasa marginal de transformación de la producción de este bien público por parte de la sociedad. (Sterner,

11 La no exclusión, es decir, no se puede prohibir a nadie el respirar el aire de una ciudad siendo el habitante de la misma.

12 Bienes libres son bienes que se consumen sin un pago que refleje su valor real.

13 Una externalidad ocurre cuando la actividad de un individuo repercute sobre el bienestar de otro, sin que se pueda cobrar un precio por ello, en un sentido positivo o negativo.

$14 \quad$ En el acápite sobre la oferta de servicios ambientales se ahonda en esta discusión

15 La regla de Samuelson para la provisión de bienes públicos se deriva de los siguientes supuestos: Se supone una función de utilidad de la sociedad, la cual tiene dos bienes uno privado (X) y otro público $(Y)$, siendo una función no decreciente en ambos bienes, con mono-tonicidad estricta. Los bienes públicos y privados se producen (o aprovechan en el caso de los bienes públicos) en base a los bienes privados, con tecnología decreciente a escala $F(Y, X)$.

La regla se optimiza a partir de la maximización del Lagrangiano:

Max_L $=\prod_{i=1}^{I} U_{i} \widehat{C Y} \pm \underset{P}{ } F(y, x)$

Desarrollando las derivadas parciales y resolviendo se obtiene la regla de Samuelson:

$\prod_{i=1}^{I} \frac{U_{i Y}^{\prime}}{U_{X}^{\prime}}=\frac{F_{X}^{\prime}}{F_{X}^{\prime}}$

Esta regla es aplicada para bienes públicos puros que no admiten congestión, se supone que todos consumimos la misma cantidad del bien, pero la valoramos distinto 
2003).

Esta regla puede responder el siguiente tipo de cuestionamientos, ¿cuántas hectáreas de bosque se estaría dispuesto a sustituir para obtener tanta cantidad de madera $u$ otros bienes provenientes del bosque? En una sociedad competitiva, las áreas forestales deberían expandirse o contraerse hasta que el valor de la sumatoria de las tasas marginales de sustitución de los bosques por otros bienes entre todos los individuos decrezca e iguale la tasa marginal de transformación de los bosques por otros bienes (Vogel, 1995).

Para el caso de la conservación de la diversidad biológica y con ella los servicios ambientales que provee, supone una política directa: se agregan todos los valores simultáneos generados por la diversidad biológica y los servicios ambientales que provee y se recomienda conservar el hábitat hasta que el costo de la última hectárea conservada iguale el valor agregado creciente.

Sin embargo, existe un problema teórico fundamental en la metodología: las preferencias son inestables a lo largo de las generaciones humanas y cualquier recomendación basada en las preferencias actuales puede dar como resultado valores subestimados de las preferencias de las futuras generaciones. (Vogel, 1995), por lo que, para mantener un mínimo de áreas que conserven la diversidad biológica, la sociedad no se debe guiar por criterios económicos sino por patrones seguros mínimos - el principio de precaución ${ }^{16}$.

¿Qué pasa con el resto de las áreas que aportan diversidad biológica y servicios ambientales a la sociedad; pero que caen fuera de las áreas de conservación prioritarias?, ¿debemos dejarlas de lado? Para este tipo de entornos que brindan servicios ambientales para la sociedad pero que están enmarcados en el manejo competitivo con otro tipo de usos del suelo, si es posible usar la regla de Samuelson.

Para ello se debe tomar en cuenta el cambio de las preferencias a través del tiempo. Esto implica que, se puede calcular cuál es el aporte de los servicios ambientales a la sociedad de estos entornos y a partir de ellos determinar qué hábitat conservar ${ }^{17}$ y en qué medida; pero sólo en forma puntual en un momento en el tiempo, ya que se debe modificar su aportación de acuerdo a los cambios en las preferencias de la sociedad.

Los propietarios del bosque y de cualquier entorno natural deciden conservar, restaurar y/o utilizar en otras actividades porciones de territorio según las preferencias y los beneficios que les aporta su entorno natural. Ya que al tomar una decisión analizan los beneficios económicos, ecológicos, sociales y culturales que les provee el entorno. Por lo que, si existe una disposición por parte de la sociedad de reconocer en forma económica los servicios prestados por el entorno natural, los propietarios del bosque tendrán un elemento adicional para analizar la conversión del bosque a otras actividades o su conservación como entorno natural.

Resumiendo, se considera que gran parte de los servicios ambientales son demandados por toda la sociedad, su conservación y manejo parte por considerar a dichos servicios como bienes públicos. Por lo cual usando la regla de Samuelson, se verá el aporte en valor de dichos servicios a la economía en general, es decir la demanda de los mismos. Partiendo de este argumento, se postularán políticas de conservación y manejo de las áreas que brindan estos servicios.

\subsection{Calculando La Disposición a Pagar por el Cambio en el uso del suelo}

Para este proceso de cálculo, se utilizará la definición de la segunda parte de la regla de Samuelson, es decir, se usará la tasa marginal de transformación de la producción de este bien público por parte de la sociedad. Es decir, se verá el aporte marginal que tienen los bienes públicos brindados por lo entornos naturales a la producción de la economía en su conjunto. Ecuación a.2, pie de página 19

Para ello se usará una técnica indirecta, la cual pasa por calcular el aporte de todo el entorno natural de una economía dentro la función de producción de la sociedad, es decir, se considera en forma explícita al territorio como insumo de la función de producción agregada ${ }^{18}$. Esto permite obtener el valor del producto marginal del entorno natural

16 Declaración sobre Medio Ambiente y Desarrollo. La Conferencia de las Naciones Unidas sobre Medio Ambiente y Desarrollo, Río de Janeiro, del 3 al 14 de junio de 1992. Principio 15: Para proteger el medio ambiente, los Estados deben aplicar ampliamente el criterio de precaución de acuerdo con sus capacidades. Cuando existan amenazas de daños graves o irreversibles, la falta de certeza científica total no se utilizará como razón para posponer medidas rentables para prevenir la degradación ambiental.

17 Esto se refiere a aquellos entornos que caen fuera del principio precautorio

18 Existe un extenso debate en la economía sobre el uso de la función de producción agregada, por

ISSN ONLINE 2521-2737| REV.INV.\&NEG. |V.14| № 23|AÑO 2021 


\title{
188 Investigación Negocios
}

\author{
$Y=f(N, P, K, S, L)_{\rceil)}$
}

Se considerara una Función de producción con tres factores productivos, capital hecho por la mano del hombre o artificial (K), los insumos naturales entendidos como el entorno en su conjunto (bosques, pasturas, tierra agrícola, etc.) (S) y la mano de obra (L) y dos indicadores que afectan la productividad de los factores, la calidad ambiental (N) y el progreso técnico (P) (Candaudap, 2019; Smulders, 2000; Solow-Stiglitz, 1997).

$P M S=\frac{\square f\left(N, P, K, S_{i}, L\right)}{\square S \quad 2)}$

Se procede a calcular la productividad marginal del entorno natural, la cual multiplicada por el producto nos da un aproximado del valor del producto marginal del entorno, el cual varía año a año según el comportamiento del producto y las preferencias de la sociedad.

Valor_P oducto_Marginal_del_entorno $=P I B \square \frac{\square\left(N, P, K, S_{i}, L\right)}{\square S}$

Se debe usar una serie de supuestos que permiten manejar en forma aproximada este fenómeno; se considera que el entorno brinda a la sociedad una serie de productos y servicios ambientales en forma agregada, por lo que en una primera etapa sólo es posible calcular el valor medio del producto marginal del entorno, que no es más que el valor marginal en forma conjunta dividido por el total de la unidad de análisis del entorno. Hay una cantidad fija de tierra pero esta tiene una demanda variable en el tiempo, la cual se observa a través del índice de productividad del territorio.

El proceso de cálculo para determinar la disposición a pagar por el mantenimiento de los servicios ambientales, partirá por determinar, mediante una función de producción agregada tipo Solow- Stiglitz ${ }^{19}$, la productividad marginal del entorno natural, la cual multiplicada por el producto nos da un aproximado del valor del producto marginal del entorno. Ponderando este valor del producto por la unidad de superficie del entorno, se obtiene el valor medio que la sociedad está dispuesta a pagar por dejar en sus actuales condiciones el presente uso del suelo.

A partir de este punto; ya que se considera al entorno como un insumo de la función de producción, la demanda particular de cada una de sus partes se debe calcular como una demanda derivada del proceso productivo de la sociedad. El aporte marginal de cada una de las partes del entorno se deduce a partir de un sistema de ecuaciones que caracteriza el comportamiento de cada uno de los tipos de coberturas vegetales, a partir de índices de producción y comportamientos en la población agrícola.

Para ello, se debe utilizar una unidad de medida del entorno natural en este caso se utilizarán unidades de superficie, hectáreas de terreno (total de la superficie del entorno), diferenciadas según el tipo de cobertura vegetal existente en el entorno de análisis, mientras mayor sea el grado de diferenciación mayor será el grado de exactitud en el cálculo del aporte de cada una de las partes ${ }^{20}$.

Aunque los actuales sistemas de contabilización de los ingresos nacionales adolecen de graves limitaciones, el PIB sigue constituyendo la base para evaluar el rendimiento económico de los diferentes sectores de la economía (FAO, 2015b). Por lo que, usar esta medida como base para la función de producción es sólo una aproximación del verdadero proceso que genera los ingresos

lo que se sugiere ver el debate en (Felipe \& McCombie, 2005)

19 Lo más que puede decirse en realidad acerca de una función de producción es que se ajusta, describe o explica más o menos bien, con mayor o menor aproximación, una determinada realidad de un sector o de una economía. Así, una industria o una economía determinada no tiene ni deja de tener una función de producción Cobb-Douglas o Solow-Stiglitz y la bondad de ese ajuste dependerá del grado en que la realidad acorde con los rasgos que caracterizan a esa función en particular.

20 Lamentablemente este tipo de bases de información suele estar limitadas por la cantidad y calidad de sus datos a través del tiempo, por lo que ésta es una limitante que hay que considerar al momento de desarrollar esta metodología. 
de un país, pero brinda insumos a partir de los cuales gobierno y sociedad generan políticas públicas de asignación de recursos.

\subsection{El modelo econométrico}

El proceso de calculó para determinar la disposición a pagar por el mantenimiento de los servicios ambientales, partirá por establecer la productividad marginal del entorno natural mediante una función de producción agregada tipo Solow-Stiglitz, con un progreso tecnológico exógeno que se observa en un cambio de productividad de territorio y de la fuerza laboral2 ${ }^{21}$, donde $K$ representa el stock de capital de la economía, medidos por la formación bruta de capital fijo- FBKF; I representa la población económicamente activa - PEA-corregida por la tasa de desempleo y multiplicado por un índice de actividad; S el entorno natural- EN- expresado en unidades de superficie ponderado por la productividad del entorno tanto agrícola como no agrícola:

$\ln (P i b)=\mathrm{b}_{0}+\mathrm{b}_{1} \ln (k)+\mathrm{b}_{2} \ln (l)+\mathrm{b}_{3} \ln (S)$

La cual está enmarcada en un sistema de ecuaciones que muestra el comportamiento del sistema de coberturas vegetales de acuerdo a las siguientes ecuaciones:

$\ln (S)=\mathrm{a}_{0}+\mathrm{a}_{1} \ln (\&)+\mathrm{a}_{2} \ln (\not \delta)+\mathrm{a}_{3} \ln (\delta)+\mathrm{a}_{4} \ln (\delta)+\mathrm{a}_{5} \ln ($ Poag $)$

Donde Sa representa la superficie agrícola, Sp la superficie de pasto permanentes, Sb la superficie boscosa, So la superficie de otro tipo de coberturas, Poag la población económicamente activa del sector agrícola.

$\ln (\mathbb{E})=\mathrm{f}_{0}+\mathrm{f}_{1}$ iagro $+\mathrm{f}_{2} \ln (\delta)+\mathrm{f}_{3} \ln ($ poagmaq $)+\mathrm{f}_{4} \ln ($ Poag $)$

Donde iagro representa en índice de productividad agrícola, y poagmaq el factor cruzado entre maquinaria agrícola y la población económicamente activa del sector agrícola.

$\ln (\not)=\mathrm{t}_{0}+\mathrm{t}_{1}$ ipastos

Donde ipastos representa el índice de productividad de la ganadería

$\ln (\mathbb{B})=\mathrm{j}_{0}+\mathrm{j}_{1}$ ibosque

8)

Donde ibosque representa el índice de productividad de los sistemas no-agrícolas

$\ln (\delta)=\mathrm{u}_{0}+\mathrm{u}_{1} \ln ($ poagsag $)$

Donde poagsag representa el factor cruzado entre la superficie agrícola y la población económicamente activa del sector agrícola

Se usaron las bases de datos del World Development Indicator y la FAO-Stat en su versión 2015, la base utilizada considera los años 1986 al 2005, se eligió como año de inicio 1986 por existir un cambio estructural significativo en la economía boliviana el año 1985, que introduce mucho ruido a la regresión. Se utilizó un sistema de cálculo de mínimos cuadrados en dos etapas y se obtuvo el siguiente conjunto de regresiones a partir del sistema de ecuaciones descrito con anterioridad, en una primera etapa desarrolla un modelo de corrección de errores que se explica y desarrolla en anexos, cuadro $N^{\circ} 5$ ver anexos. El método de mínimos cuadros en dos etapas, permite corregir problemas de multicolinealidad en el sistema de ecuaciones, así como desarrollar una estimación consistente que da una inferencia que usa las interrelaciones del sistema de ecuaciones.

$21 \quad$ Modelo visto en Smulders, donde se ejemplifica un modelo con tecnología exógena, donde

$Y=(l * L)^{\mathrm{a}} *(K)^{\mathrm{b}} *(t * T)^{1\lceil\mathrm{a} \square \mathrm{b}}$ el cambio tecnológico esta dado por índices de productividad que afectan a la mano de obra y el uso del suelo 


\section{Investigación $\&$ Negocios}

\section{Cuadro $\mathrm{N}^{\circ} 3$}

\section{Elasticidad media por Tipo de Cobertura Vegetal}

\begin{tabular}{|c|c|c|c|c|c|}
\hline $\begin{array}{c}\text { Superficie } \\
\text { Total }\end{array}$ & Arable & $\begin{array}{c}\text { Pasturas Per- } \\
\text { manentes }\end{array}$ & Forestal & $\begin{array}{c}\text { Otro Tipo de Co- } \\
\text { bertura }\end{array}$ & \\
\hline 0.14937 & 0.01743 & 0.05128 & 0.05133 & 0.00814 & 0.0212 \\
\hline
\end{tabular}

Si bien el modelo permite discutir más allá de la rentabilidad media del tipo de cobertura vegetal, adentrarse en esta discusión saca de análisis el tema en debate, es decir, el pago de servicios ambientales mediante el reconocimiento como insumo productivo de los servicios conexos que brinda cada tipo de vegetación en especial las coberturas boscosas y arbustivas. Mediante un pequeño cálculo, se puede recuperar la elasticidad de cada una de las coberturas vegetales mediante la multiplicación de las elasticidades parciales, cuadro $N^{\circ} 3.22 \mathrm{~A}$ su vez, utilizando el concepto de valor del producto marginal visto en la sección anterior, se puede hallar el valor medio que recibe la sociedad por cada tipo de cobertura. Cuadro $N^{\circ}$ 4. La cual muestra la elasticidad media de cada una de las coberturas multiplicada por el producto de toda la economía

\begin{tabular}{|c|c|c|c|c|c|}
\hline \multicolumn{6}{|c|}{ Cuadro $N^{\circ} 4$} \\
\hline \multirow{2}{*}{\multicolumn{6}{|c|}{$\begin{array}{c}\text { Aporte Medio por Tipo de Cobertura Vegetal } \\
\text { Expresado en dólares americanos }\end{array}$}} \\
\hline & & & & & \\
\hline Año & Global & Arable & Pasturas Permanentes & Forestal & $\begin{array}{c}\text { Otro Tipo de Co- } \\
\text { bertura }\end{array}$ \\
\hline 1986 & 5.45 & 31.31 & 6.15 & 3.58 & 5.61 \\
\hline 1987 & 5.99 & 34.02 & 6.76 & 3.95 & 6.02 \\
\hline 1988 & 6.33 & 35.61 & 7.14 & 4.19 & 6.22 \\
\hline 1989 & 6.50 & 36.20 & 7.28 & 4.31 & 6.44 \\
\hline 1990 & 6.70 & 37.62 & 7.52 & 4.46 & 6.46 \\
\hline 1991 & 7.36 & 40.56 & 8.18 & 4.91 & 7.31 \\
\hline 1992 & 7.77 & 42.19 & 8.64 & 5.21 & 7.42 \\
\hline 1993 & 7.90 & 42.03 & 8.69 & 5.32 & 7.68 \\
\hline 1994 & 8.24 & 41.43 & 9.07 & 5.57 & 7.84 \\
\hline 1995 & 9.25 & 43.91 & 10.18 & 6.29 & 8.64 \\
\hline 1996 & 10.19 & 46.82 & 11.21 & 6.96 & 9.24 \\
\hline 1997 & 10.92 & 45.58 & 12.01 & 7.50 & 9.91 \\
\hline 1998 & 11.71 & 47.21 & 12.88 & 8.08 & 10.37 \\
\hline 1999 & 11.41 & 45.58 & 12.56 & 7.92 & 9.76 \\
\hline 2000 & 11.56 & 46.70 & 12.72 & 8.06 & 9.46 \\
\hline 2001 & 11.05 & 45.09 & 12.16 & 7.74 & 8.69 \\
\hline 2002 & 10.75 & 43.77 & 11.82 & 7.55 & 8.27 \\
\hline \multicolumn{6}{|c|}{ Elaboración propia } \\
\hline
\end{tabular}

Todo este proceso de cálculo nos permite revelar en forma indirecta el monto que la sociedad recibe por tener las actuales coberturas del suelo en Bolivia, monto que puede equipararse según la ecuación de Samuelson a la disposición a pagar por conservar el actual estado de conservación de 22 El cálculo se realiza multiplicando las elasticidades parciales estimadas en los cuadros $N^{\circ} 5$ y 7 
la cobertura vegetal. De estas disposiciones a pagar es prioritario darle énfasis a dos, la primera el pago a la conservación de la cobertura boscosa y la segunda a la conservación de otro tipo de coberturas, en especial sistemas de matorrales. Estos dos tipos de coberturas con sus respectivos ecosistemas son en gran medida los proveedores de una multiplicidad de servicios ambientales que aún no son reconocidos por la sociedad.

El reconocer este aporte en forma monetaria, brindará la oportunidad a la sociedad en su conjunto, a los administradores y propietarios del bosque y de los sistemas de matorrales de reevaluar la conservación y manejo de este sistema, al generar una política que posibilite realizar este pago a los administradores y gestores de estos sistemas, las comunidades originarias y los sistemas de áreas protegidas.

\section{Entre la Oferta y la Demanda: Propues- ta de Política Pública para el Manejo de Servicios Ambientales}

Desde el momento en que reconocemos la existencia de un bien público, y observamos que existe una tendencia a su pérdida o deterioro, es necesario definir una intervención para corregir la falta de reconocimiento $-\mathrm{y}$ asignación de recursos- del mercado para garantizar la adecuada provisión de estos servicios a la sociedad.

Si identificamos una externalidad derivada de la actividad de un individuo, (el propietario o "cuidador" del bosque), que repercute sobre el bienestar de otro (la sociedad en su conjunto a través de su impacto en la actividad económica nacional), sin que se pueda cobrar un precio por ello, estamos ante una falla de mercado que requiere la intervención del Estado.

La intervención puede tomar la forma de:

- Retribución. Cuando se busca resarcir al propietario por el costo incurrido en la producción del SA.

- Compensación. Cuando se realiza un pago equivalente al ingreso perdido por el productor del SA (Costo de oportunidad)

- Subsidio. Cuando se realiza una transferencia de recursos públicos a través de una inversión o mediante un pago directo, como un incentivo al productor del SA para evitar la pérdida de un bien público.
- Pago. Cuando se reconoce (y valoriza) la producción de un servicio ambiental como resultado de una actividad "productiva", reconociendo al productor los costos directos e indirectos, el riesgo y su legítimo derecho a una utilidad (Excedente del productor).

Al tratarse de un bien público que exige la intervención del Estado, éste representa a la demanda. El proceso de decisión del demandante es equivalente al del propietario del bosque, u oferente, descrito líneas arriba.

- Identificación de los conjuntos de oportunidades

- Definición de las disyuntivas

- Cálculo correcto de los costos

En el análisis de las oportunidades deberá identificar su (s) segunda (s) mejor opción: ¿Existen otras fuentes potenciales? ¿Qué representaría no lograr el propósito de la intervención? ¿Cuál es el costo para la sociedad de perder estos servicios ambientales?; ¿Cuál es el costo de reposición, si esta posibilidad existe? y finalmente; ¿Cuál es el costo en que incurre el productor del servicio por producir, mantener el servicio o dejar ir otras oportunidades? Aquí cobra relevancia entender el marco de preferencias y desarrollar los cálculos de los beneficios monetarios y no monetarios que se le brinda a la sociedad por mantener las actuales coberturas vegetales

Al igual que cualquier consumidor, cuando el Estado representa la demanda, enfrenta siempre una restricción de presupuesto: Cualquier decisión sobre el uso de los recursos públicos competirá con otros objetivos de política y la provisión de bienes y servicios públicos igualmente importantes.

Dentro del contexto de la economía boliviana, el manejo y conservación de recursos naturales puede estar regido por una lógica productiva y/o por la mayor certeza en la tenencia de la tierra:

- En el primer caso, prevalecen las preferencias económicas por sistema de generación de recursos priman en los procesos productivos ${ }^{23}$.

- El segundo responde al sistema de exclusión en los sistemas de producción relacionados al mercado y el mal reparto de tierras de Bolivia, que pueden llevar a que las lógicas de des- 


\section{Investigación \& Negocios}

trucción de la tierra primen sobre los proceso de conservación de territorios no aptos para la producción agrícola y ganadera, ya que para asegurar la propiedad de la tierra, se procede a la destrucción de la cobertura vegetal original; respondiendo al concepto de que la tierra es para quien la trabaja y la conservación no representa trabajo para este tipo de lógica.

Ambos procesos, se evidencian en la reducción de las superficies boscosas del país; pero desde diferentes sentidos: el primero desde el objetivo de producir alimentos o generar recursos económicos vía la producción agrícola, forestal y ganadera, la segunda desde la necesidad de afianzar los derechos de propiedad de un territorio.

El pago de servicios ambientales, trata de encarar ambas caras de esta moneda

- Primero, se parte del reconocimiento de los servicios ambientales prestados por las coberturas vegetales, por lo que, se hace necesario el pago del servicio a los poseedores y administradores de este territorio vía el cobro de los servicios a toda la sociedad. Este pago hace que los poseedores del territorio antes de realizar un cambio en el uso del suelo tengan un elemento adicional en su estructura de costos que les permita enfrentar el beneficio de conservar un sistema boscoso o dedicar el territorio a una actividad productiva.

- Segundo el instrumento de hecho reconoce el derecho de propiedad de la comunidad o de los poseedores del territorio, sin la necesidad de desarrollar actividades productivas en suelos no aptos para este tipo de procesos productivos.

El pago de los servicios ambientales tradicionalmente parte por reconocer los costos de oportunidad medios de los productores, este pago pretende que no se utilicen las coberturas boscosas existentes en procesos productivos tradicionales, como la agricultura y la ganadería. En este caso proponemos que el pago del servicio ambiental sea la valoración media que tiene la sociedad sobre las áreas boscosas ${ }^{24}$ y sea el productor quien decida si realizar o no el cambio en el uso del suelo. Así la elección del productor depende del comportamiento de la economía en su conjunto (sobre el que se calcula el pago), del marco de preferencias del productor y de los costos de oportunidad personales dadas sus actividades productivas.

La propuesta de política pública realizada en este trabajo parte de:

- El pago de servicios ambientales, debe ser un pago por actividades de conservación ${ }^{25}$ y manejo del bosque y no así un subsidio.

- Debe existir una cobertura mínima de bosque por el cual se realiza el pago, la cual debe determinarse por criterios biológicos de acuerdo al piso ecológico donde se ubique.

- El pago se realizará a predios en los cuales, no se realicen actividades que vayan en contra de la producción de los servicios ambientales del bosque, como ser los aprovechamientos forestales no sustentables, y los cambios de uso del suelo forestal para el establecimiento de plantaciones destinadas producción maderera y para habilitar terrenos dedicados a la actividad agrícola y ganadera.

- Las áreas sujetas a este pago, se encontrarán en las partes altas de las cuencas hidrográficas pobladas por un número de habitantes mínimo para ser consideradas pequeñas ciudades intermedias (INE, 2002) ${ }^{26}$, no pudiendo cubrir este beneficio más de un porcentaje del territorio Boliviano. Dicho porcentaje debe ser calculado de acuerdo a las limitaciones presupuestarias del programa que se realice de acuerdo a esta propuesta.

- Los grandes beneficiarios de esta política son las comunidades campesinas y las áreas protegidas nacionales, prefecturales y municipales. Adicionalmente se puede considerar el pago a áreas privadas siempre y cuando cumplan con los criterios antes mencionados y se encuentren en regiones no aptas para el desarrollo de otro tipo de

\footnotetext{
$24 \quad$ Obtenida mediante el producto interno bruto de la economía y la rentabilidad media de cada tipo de cobertura vegetal

25 Según el piso ecológico estas actividades van desde la creación de terracería para evitar erosión del terreno creación de corta incendios, manejo de plagas, reforestación, control de incendios, etc.

26 En Bolivia existen aproximadamente 75 poblaciones con más de 5,000 habitantes.
} 
actividades productivas.

- El pago de los servicios ambientales, se realizaría mediante el cobro de un derecho, a todos los beneficiarios de los servicios a nivel nacional de acuerdo a los criterios a ser mencionados en el siguiente párrafo.

Se propone realizar el cobro de este servicio, a través de las tarifas de consumo de agua potable, energía eléctrica y combustibles, con una tarifa progresiva según la intensidad de uso de estos servicios, quedando exento de pago una cantidad mínima del uso de agua potable y energía eléctrica, por ser consideradas por la sociedad boliviana como un derecho inalienable.

El financiamiento de las intervenciones propuestas puede realizarse a través de un impuesto a aquellas actividades que generan impactos negativos al ambiente y/o usen los servicios ambientales como insumo productivo, ya sea en el proceso mismo de su producción o como resultado del uso del bien producido. Este es el caso del agua y la energía: desde el proceso de extracción y transformación hasta su uso e impacto ambiental derivado, por descargas o consumo. Ello a través de un impuesto recaudatorio y modificatorio de conductas. A este tipo de impuesto, se le denomina impuesto Pigouviano y tiene un doble propósito (no siempre fácil de balancear).

- Recaudatorio. Para financiar las actividades de manejo y conservación de los recursos naturales y "premiar" a los propietarios o cuidadores del bosque.

- Modificatorio de conductas. Para generar incentivos, positivos o negativos, que inclinen el terreno en favor de las conductas deseables o en detrimento de aquellas que se pretende eliminar o reducir.

La ventaja de este sistema de cobro es que se realizará por intensidad de uso, por lo que los usuarios con mayores ingresos y mayor intensidad de uso serán las que paguen un mayor porcentaje del programa. Mediante una simulación que considera el cobro medio de servicios ambientales por familia para el año 2002, este no excedería los tres dólares americanos por mes (aproximadamente 25 Bs.). Se desarrolla la simulación usando los datos del cuadro $\mathrm{N}^{\circ} 4$, con una cobertura de pago del $25 \%$ del territorio ocupado principalmente por sistemas boscosos. Como se observa, se puede incluir una cantidad importante de territorio dentro el pago de servicios ambientales sin que ello represente un incremento sustancial en el gasto familiar, ver cuadro $\mathrm{N}^{\circ} 6.2$.

El pago de servicios ambientales tendrá como principales beneficiarios a las comunidades campesinas y los habitantes de las áreas urbanas y peri urbanas, los primeros por la generación de una nueva fuente de ingresos que les permitirá conservar y mantener en mejores condiciones sus recursos naturales, siendo incluidos en el sistema económico vigente al ser reconocidos como los principales proveedores de insumos productivos indirectos como lo son los servicios ambientales; y los segundos por contar con el mantenimiento y conservación de sus principales fuentes de agua potable y servicios ambientales en general que van en beneficio directo de todo el sistema productivo, ver cuadro $N^{\circ} 6.11$.

\section{Conclusiones.}

Dadas la actuales condiciones políticas de Bolivia, teniendo en puerta el desarrollo de una nueva constitución política del Estado Boliviano, es necesario introducir en la discusión el concepto de servicios ambientales, pero no sólo como una herramienta de conservación y manejo del bosque, sino como una herramienta integral que permite afrontar los conflictos ambientales y los conflictos sociales que aquejan al país. Esto por la inequidad en el acceso a los recursos y el no reconocimiento de los aportes que realizan las comunidades campesinas a la productividad de toda la sociedad, en especial a los sistemas agropecuarios y a los centros urbanos, al conservar grandes extensiones de bosques y tierras arbustivas.

El trabajo parte por definir y ejemplificar los procesos de pagos de servicios ambientales para luego introducir la necesidad de reconocer como sociedad la importancia de manejar en forma integral la conservación del bosque pasando más allá de los conceptos de conservación para llegar a los aportes de los ecosistemas a la productividad de un territorio. Si se quiere conservar esta productividad es necesario conservar y manejar los recursos que la generan.

Las propuestas de política realizadas en el trabajo parten de un análisis exhaustivo de las lecciones dadas por otros sistemas de pagos de servicios ambientales en la región latinoamericana y en el mundo, corrigiendo y modificando las visiones con las que fueron crea- 


\section{Investigación \& Negocios}

dos para adecuarlas a la compleja realidad boliviana.

La principal lección que obtuvimos de este proceso de investigación es que un pequeño ajuste en el sistema de derechos por el uso de agua potable, energía eléctrica y uso de combustible, puede ayudar a conservar y manejar en forma eficiente y sostenible en el tiempo, vía el pago de los servicios ambientales, una porción importante del territorio boliviano en manos de las comunidades indígenas y campesinas.

Si bien éste es un tema nuevo en la discusión académica, social y política en Bolivia es necesario afrontarla desde diferentes perspectivas, siendo este trabajo una de estas visiones, la cual busca principalmente poner sobre la mesa el tema a discusión y avanzar en el desarrollo del mismo.

\section{Referencias}

- Azqueta Diego. (2012). "Valoración económica de la calidad ambiental” Editorial: Mc Graw-HiII. Madrid.

- Candaudap Luis Eduardo. (2019). Repercusiones de la Calidad Ambiental en el crecimiento económico. Formulación y obtención de un índice de calidad ambiental y sus efectos en la función de producción para el caso de México. Universidad Nacional Autónoma de México. México.

- Carvajal, M. J. M., González, A. B., Bontemps, L. C., López, S. V., Bello, G. M. C., \& Juárez, J. R. (2019). Pago por servicios ambientales hidrológicos y dinámica de la cobertura arbórea en la región Iztaccíhuatl-Popocatépetl, Puebla. Acta Agronómica, 68(2).

- Declaration on Environment and Development. (1992). The United Nations Conference on Environment and Development, Rio de Janeiro from 3 to 14 June.

- Felipe J. \& McCombie J.S.L.. (2005). La Función de Producción Agregada en Retrospectiva. Investigación Económica. Julio - Septiembre, pp. 43-88. Vol. 64.

- $\quad$ Freeman, A. M.. (1993). The measurement of environmental and resource values, Resources for the Future, Washington, DC.

- Jackson Robert B., Esteban G. Jobba 'gy, Roni Avissar, Somnath Baidya Roy, Damian J. Barrett, Charles W. Cook, Kathleen A. Farley, David C. le Maitre, Bruce A. McCarl, Brian C. Murray. (2005). Trading Water for Carbon with Biological Carbon Sequestration. Science, December 23. pp. 1944-1947. Vol. 310

- Landell-mills Natasha \& Ina T. Porras. (2002). Silver bullet or fools' gold?. International Institute for Environmental Development, London.

- Madrid, Sergio editor. (2016). Estudio de casos. Consejo Civil Mexicano de Silvicultura Sustentable, México. (inédito)

- Mabsout, R., \& Radmard, H. (2019). Experimenting with the Coase theorem. Journal of Economic Methodology, 1-17.

- Miranda Miriam. (2003). The social impacts of payments for environmental services in Costa Rica. A quantitative field survey and analysis of the Virilla watershed. International Institute for Environmental Development. UK.

- Muñoz Piña, Carlos, Alejandro Guevara, Juan Manuel Torres and Josefina Braña. (2005). Paying for the Hydrological Services of Mexico's Forests: analysis, negotiations and results. INE, Mexico.

- Pagiola S, Bishop J. \& Landell-Mills N. (2003). La Venta de Servicios Ambientales Forestales. Instituto Nacional de Ecología. México.

- Sterner, Thomas. (2003). Policy Instruments for Environmental and Natural Resource Management. Resources for the Future Press, Washington, D.C. 
- Organización de las Naciones Unidas para la Agricultura y la Alimentación - FAO (a). (2015). Situación de los Bosques del Mundo. Italia.

- $\quad$ Organización de las Naciones Unidas para la Agricultura y la Alimentación - FAO (b). (2015). FAO Statistical Databases. Italia.

- Rosas-Baños, M. (2013). Nueva Ruralidad desde dos visiones de progreso rural y sustentabilidad: Economía Ambiental y Economía Ecológica. Polis. Revista Latinoamericana, (34).

- Rosa Herman, Susan Kandel \& Leopoldo Dimas. (2003). Compensation for Environmental Services and Rural Communities. Lessons from the Americas and Key Issues for Strengthening Community Strategies. PRISMA, San Salvador.

- Sanjines Esteban. (2005). la aplicación del proceso de reforma agraria en Bolivia. Agro Nuevo. Número 7. Julio. pp. 39-56.

- Smulders, Sjak. (2000). "Econometric Growth and Enviromental Quality", en Elgar,Edward, Principles of Enviromental Economics, Henk Folmer, and Landis Gabel editores

- Solow R. M. (1997). "Georgescou-Roegen versus Solow/Stiglitz. Reply", en Ecological Economics, $N^{\circ} 22$, pp. 267-268.

- Vogel, Joseph H. (1995). A market alternative to the valuation of biodiversity: the example of Ecuador. Association of Systematics Collection Newsletter October: 66-70. 